\title{
Ultrasound Imaging using Laser Induced Breakdown
}

$\begin{array}{lll}\text { Member } & \text { Osamu Oshiro } & \text { (Nara Institute of Science and Technology) } \\ \text { Non-member } & \text { Atsunori Machida } & \text { (Nara Institute of Science and Technology) } \\ \text { Non-member } & \text { Motonori Doi } & \text { (Nara Institute of Science and Technology) } \\ \text { Non-member } & \text { Kunihiro Chihara } & \text { (Nara Institute of Science and Technology) } \\ \text { Non-member } & \text { Daisuke Ueda } & \text { (Osaka University) } \\ \text { Non-member } & \text { Tadao Sugiura } & \text { (Osaka University) } \\ \text { Non-member } & \text { Satoshi Kawata } & \text { (Osaka University) }\end{array}$

Keywords: instantaneous imaging, ultrasound, laser induced breakdown (LIB), synthetic aperture method, spherical wave

\section{Introduction}

Ultrasound (US) imaging is widely used because it visualizes the exterior structure of a measurement object and the interior structure that cannot be visualized with an optical measurement method. Therefore, it is an essential tool as non-destruction test of a steel pipe and a railway and so on in the industrial field and as diagnosis of a human organ like a heart, a liver and so on in the medical field. Furthermore, US imaging is also used in the fishery or military field.

In the conventional US imaging method, a US beam scans the object electrically to reconstruct a twodimensional (2D) image ${ }^{(1)}$. The image has about the frame rate of 10-100 fps (frame per second) but it is difficult to increase the frame rate of the image because ultrasound has the finite velocity in a media, for example, $1,500 \mathrm{~m} / \mathrm{sec}$ in water. In the last decade, the research on three-dimensional (3D) ultrasound imaging is often discussed ${ }^{(2)}$. However, it takes a longer time to reconstruct a $3 \mathrm{D}$ image because of the finite velocity of ultrasound and the frame rate is below $10 \mathrm{fps}$.

We have been studying the instantaneous US imaging system ${ }^{(3)}$, where pulsed US with little directivity was transmitted once, the wave reflected from a measurement object was received at several points and the $3 \mathrm{D}$ image of a measurement object was reconstructed with the synthetic aperture method ${ }^{(4)}$. As only one transmission of ultrasound enables to reconstruct a $3 \mathrm{D}$ image this imaging method has the potential to obtain a $3 \mathrm{D}$ image with frontally wide view and very high frame rate above 1,000 fps. However, the following problems are encountered;

(1) The transmitted waveform has not single peak but many peaks owing to the transducer ringing on transmitting ultrasound (ringing phenomenon), which results in blurring the reconstructed image.

(2) Ultrasound with low directivity cannot be transmitted from a transducer of a piezoelectric device, for example, made of ceramics. The ultrasound enables to visualize only the very narrow region.

Judging from the the matters mentioned above, the instantaneous US imaging is waiting for the pulsed ultrasound with low directivity.

When pulsed laser beam was radiated to atoms or molecules in the very small region, some atoms or molecules absorb light, which induces to raise the energy level and to ionize the atoms or molecules. This ionization causes a cascade ionization of other atoms or molecules. Then, ionized atoms or molecules are recombined with an electron. Immediately, light emission, shock wave and cavitation follows. Consequently, light, heat and sound are generated from the laser focal point. This phenomenon is called 'laser induced breakdown (LIB) ${ }^{(5)}$. As the sound is generated from a very small source, it is thought to have the low directivity. Therefore, at first, we performed the generation of ultrasound from LIB and examined the characteristics of the ultrasound. Next, we carried out the instantaneous imaging using the ultrasound and the synthetic aperture method. This paper describes the experiment to generate ultrasound from LIB, the investigation of the waveform and directivity of the ultrasound and the reconstructed 3D image using this method.

\section{Laser Induced Breakdown}

First, we performed the experiment to generate ultrasound using LIB in water. Table 1 summarizes the parameters of the pulsed laser for generating ultrasound.

The pulsed laser beam was focused in a water bath using an optical lens with the focal length of $30 \mathrm{~mm}$. Figure 1 is the photograph of the LIB phenomenom recorded with a digital video camera. We recorded the LIB phenomenom with the camera and confirmed that LIB generated at the almost same position. In this figure, the LIB occurred at the bright spot and heat was 
Table 1. The parameters of pulsed laser for generating ultrasound.

\begin{tabular}{|l|l|}
\hline pulsed laser & Nd-YAG \\
\hline wavelength & $1064 \mathrm{~nm}$ \\
\hline pulse repetition frequency & $10 \mathrm{~Hz}$ \\
\hline pulse energy & $10 \mathrm{~mJ}$ (typ.) \\
& $0.2 \times 10^{9} \mathrm{~J} / \mathrm{m}^{2}$ \\
& $1.25 \times 10^{6} \mathrm{~W}$ \\
\hline pulse width & $8 \mathrm{nsec}$ \\
\hline laser beam radius & $16 \mathrm{~mm}$ \\
\hline focused laser beam radius & $16 \mu \mathrm{m}$ \\
\hline focal length of the lens & $30 \mathrm{~mm}$ \\
\hline
\end{tabular}

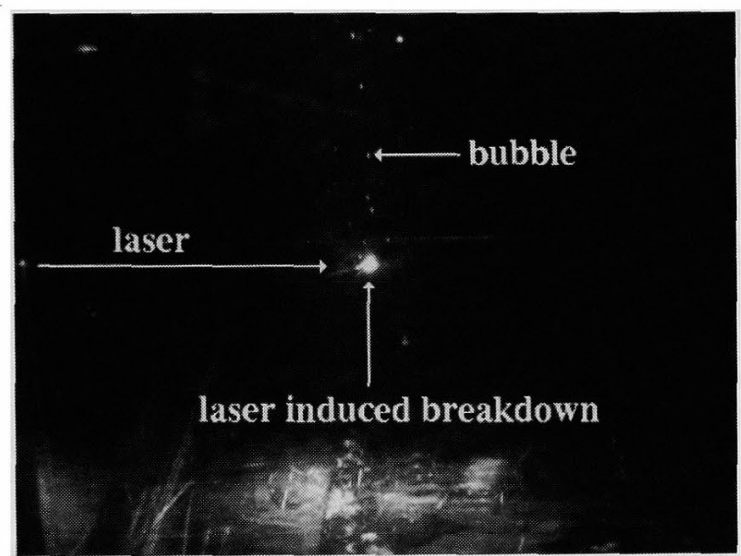

Fig. 1. Laser induced breakdown phenomenon in a water bath. The LIB occurred at the bright spot and heat was thought to be generated because of bubble generation.

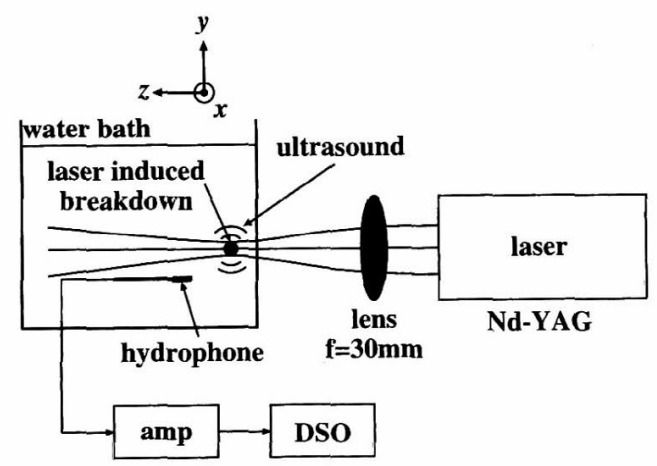

Fig. 2. The directivity measurement arrangement (the result is shown in fig. 4). The $z$ direction is set on the laser beam, and the others are normal to the $z$ direction. Using a needle hydrophone made of polymer, ultrasound was received, amplified and stored in a digital storage oscilloscope (DSO).

also generated because bubbles were thought to be produced with water boiling. Furthermore, we can hear the click-like sound with this phenomenon.

Second, we examined the waveform of the ultrasound that was generated from LIB. Figure 2 is the experimental arrangement to observe the waveform of the ultrasound. The $z$ direction is set on the laser beam, and the others ( $x$ and $y$ ) are normal to the $z$ direction; $x$ is horizontal and $y$ is vertical. This time, ultrasound

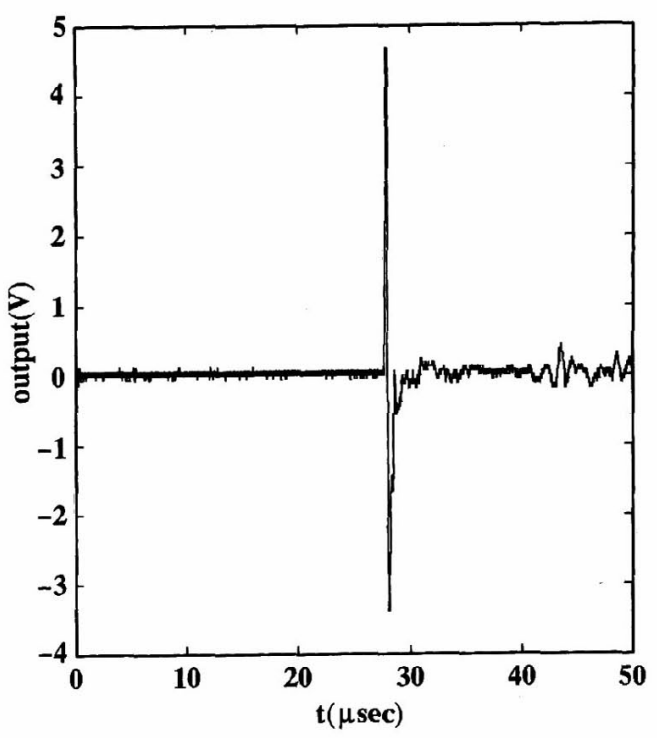

Fig. 3. The example of the waveform of the ultrasound. The waveform has the large peak at $30 \mu \mathrm{sec}$ after LIB and small ones in the range of 30-50 $\mu$ sec. The former is the wave received from LIB point directly and the latter is reflected at the wall of a water bath or the water surface.

was received with a needle hydrophone made of polymer because it has the higher sensitivity than a receiver made of ceramics. The received signal was amplified and stored in a digital storage oscilloscope (DSO) at the sampling frequency of $500 \mathrm{MHz}$.

Figure 3 is the example of the waveform of the ultrasound. In this measurement, pulsed laser was radiated at the time of 0 . As the lag time between laser radiation and LIB was very small (a few nano second), the ultrasound could be considered to be generated at the time of 0 . The wave has a large and sharp peak at $30 \mu \mathrm{sec}$ after LIB occurred and the waveform is the sinusoid wave with the period of 200 nsec. Besides, the small peaks appear in the range of $30-50 \mu \mathrm{sec}$.

The former peak is the wave received from LIB point directly. This result demonstrates that we succeeded in transmitting pulsed ultrasound and that is one characteristic for the desired ultrasound. As the pulsed laser was radiated in this measurement, this figure shows the impulse response of the imaging system including the hydrophone and the amplifier.

On the other hand, the latter peaks are the waves reflected at the wall of a water bath or the water surface and the period of the wave become larger as reflected waves from various points were superimposed.

Next, we investigated the directivity of the ultrasound generated from LIB. In this experiment, the ultrasound was received at about 100 points. The measurement points were varied at the constant step of $10 \mathrm{~mm}$ in the $x, y$ and $y$ directions. The points were moved from -70 to $70 \mathrm{~mm}$ in the $x$ direction, while from 0 to $70 \mathrm{~mm}$ in the $y$ and $y$ directions owing to the finite volume of the water bath. On the $z$ axis, the hydrophone was not set 


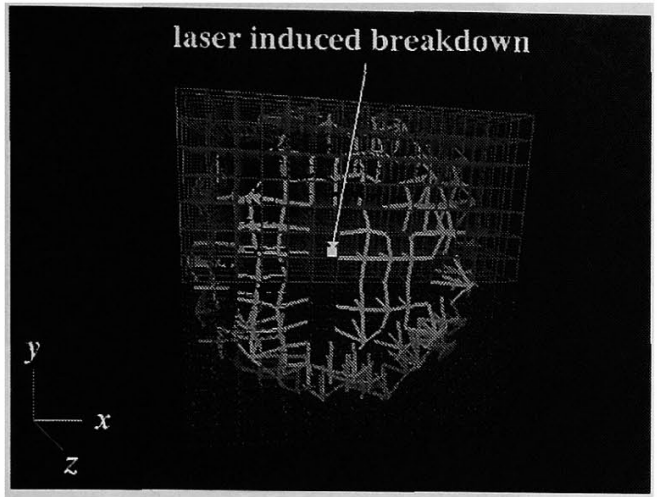

(a)

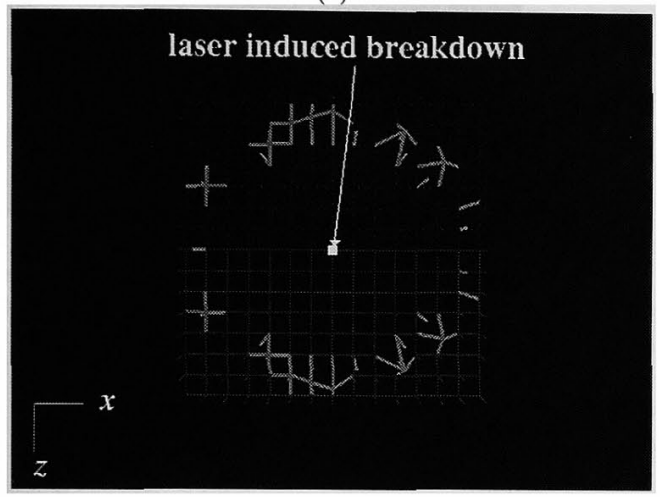

(b)

Fig. 4. The propagation of ultrasound at the time of $270 \mu \mathrm{sec}$ after LIB, (a) three dimensional image and (b)two dimensional image on the plane with $y$ $=10 \mathrm{~mm}$. We assumed that the ultrasound propagation is symmetry in the $y$ and $z$ directions. The measured points were drawn in a bright and solid line and the others in a dark and dotted line. Nodes of the grid correspond to the measurement points. In this figure, the grid was distorted as the node were shifted in proportion to the amplitude of signal and the nodes were drawn brightly.

to avoid the damage by laser.

Figure 4 shows the propagation of ultrasound at the time of $270 \mu \mathrm{sec}$ after LIB, (a) three dimensional image and (b)two dimensional image on the plane with $y$ $=10 \mathrm{~mm}$. We assumed that the ultrasound propagation is symmetry in the $y$ and $z$ directions as reported previously ${ }^{(5)}$. The measured points were drawn in a bright and solid line and the others in a dark and dotted line. Nodes of the grid correspond to the measurement points. In this figure, the grid was distorted as the nodes were shifted in proportion to the amplitude of signal received at the measurement point and the nodes were drawn brightly.

This figure reveals that the ultrasound propagated toward any direction. Therefore, we succeeded in transmitting the spherical wave and that is the other characteristic for the desired ultrasound.

\section{Ultrasound Imaging}

Here, we carried out the instantaneous imaging using the ultrasound generating from LIB. In our experiment,

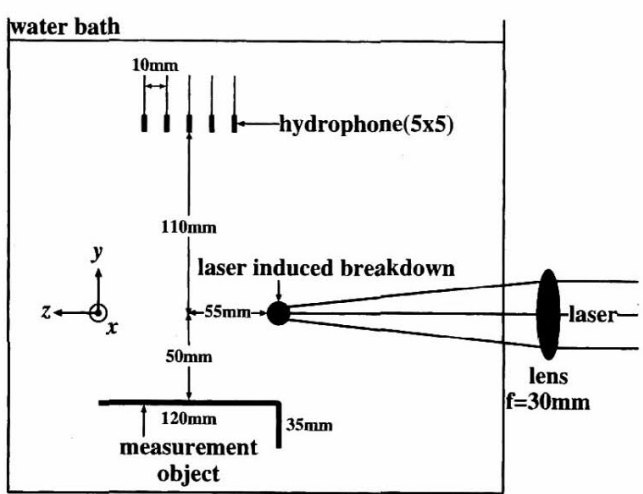

Fig. 5. The instantaneous imaging arrangement The measurements were carried out 25 times done in water and the waves were also measured at 25 points moving the hydrophone at the constant step of $10 \mathrm{~mm}$.

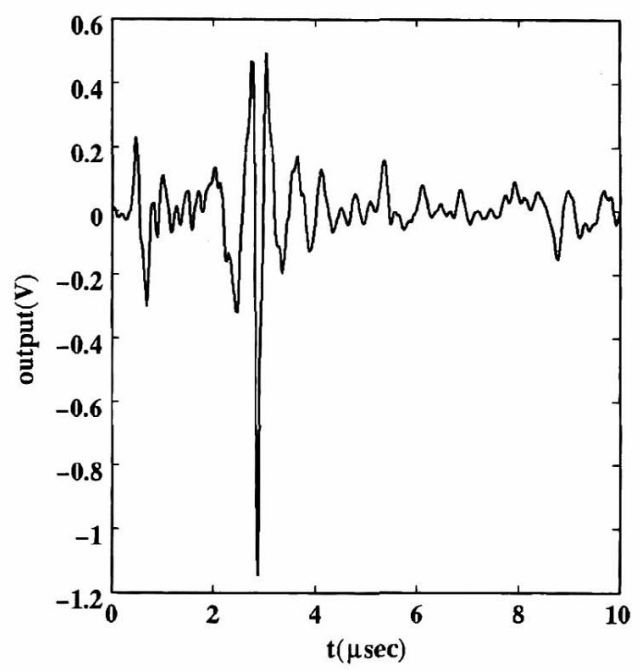

Fig. 6. The example of the reflected wave. This waveform has more peaks, which indicates that reflected signals were received from various portions of a measurement object.

we intended to confirm that ultrasound from LIB can be used for the imaging. Therefore, a steel wrench was selected as a measurement object so that the signal with larger amplitude can be received. This imaging method has the potential to obtain a 3D image with frontally wide view and we selected a long object. In this experiment, LIB was generated in a water bath and a measurement object and a hydrophone were arranged in the same bath as shown in fig. 5 .

The reflected wave from the target object was received at 25 points on the $x-z$ plane, which was $110 \mathrm{~mm}$ apart from LIB point and $160 \mathrm{~mm}$ from the target object. In this experiment, the measurements were carried out 25 times moving the hydrophone at the constant step of $10 \mathrm{~mm}$ because the measurement object does not move. The amplified signal was sampled at the frequency of $500 \mathrm{MHz}$ and stored in a wave memory. 
Figure 6 shows the reflected waveform measured at the center of 25 hydrophones. In this experiment, the ultrasound was also generated at the time of 0 . In comparison with fig. 3 , this waveform has more peaks, which indicates that reflected signals were received from various portions of a measurement object.

After measurement, received signals were sent to a personal computer and 3D image reconstruction was performed using the synthetic aperture method ${ }^{(4)}$. In our experiment using this method, a 3D object was imaged as follows;

(1) A sound source, for example an ultrasound transmitter, a measurement object and several ultrasound receiver are positioned in water.

(2) A limited measurement region is determined and divided into small elements (voxel).

(3) Ultrasound fight time from a sound source to a receiver by way of an element is calculated for all receiver and elements.

(4) Pulsed and spherical ultrasound is transmitted once.

(5) The wave reflected from a measurement object is received at several receiver.

(6) Magnitude of echo signal from an element are summed for all receiver.

(7) Summed values are converted to gray level 0255.

(8) The 3D image of a measurement object is reconstructed.

Figure 7 shows the example of 3D image, where (a) and (b) are the images observed from the different directions. The insets of this figure are the photographs of the measurement object. The measurement and reconstruction of an image took about $0.1 \mathrm{sec}$. Comparing the $3 \mathrm{D}$ images with the insets in fig. 7 , the $3 \mathrm{D}$ image can be reconstructed correctly, especially the corner of the measurement object can be visualized as indicated by the arrow shown in fig. 7 (b).

The results indicate that the ultrasound generated from LIB can be used for the instantaneous imaging. In comparison with fig. 7 (a), fig. 7 (b) shows low SN (signal to noise) ratio. As seen in the experimental setting (fig. 5), the shorter bar of a wrench was located in far side of LIB point, which results in reduction SN ratio. The ultrasound from LIB does not show ringing phenomenon. Therefore, it has the possibility to obtain the higher resolution image than the piezoelectric transducer which shows the ringing phenomenon.

\section{Conclusion}

We performed the generation of ultrasound from LIB and the instantaneous imaging using the ultrasound. We succeeded in generating pulsed ultrasound and confirm that the propagation of ultrasound shows little directivity. Using the ultrasound, we carried out instantaneous imaging and the 3D image was reconstructed very correctly. The experimental results indicate and that the ultrasound from LIB is promising for the instantaneous imaging. Moreover, the characteristics of the ultrasound, no directivity and no ringing phenomenon,

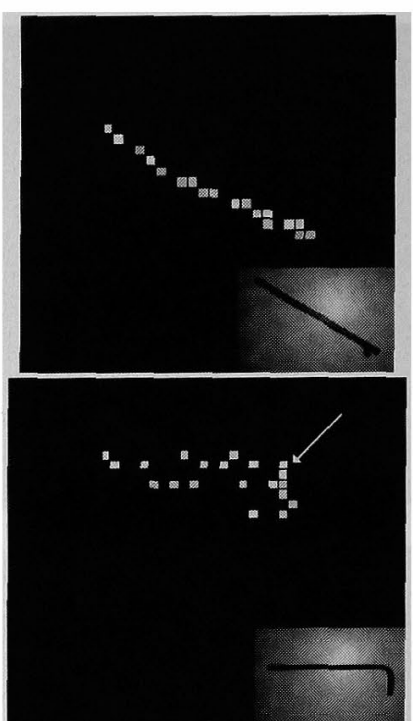

(a)

(b)

Fig. 7. The examples of the reconstructed image. where (a) and (b) are observed from the different directions. The insets are photographs of a measurement object viewed from the same direction as the reconstructed image.

reveal the potential for higher resolution imaging.

This work was partially supported by JSPS Research for the Future Program (JSPS-RFTF97I00402).

(Manuscript received June 16, 2000, revised March 5, 2001)

\section{References}

(1) K. K. Shung and M. B. Smith, "Principles of Medical Imaging", Academic Press, California. pp.78-163, 1992.

(2) A. Fenster and D. B. Downey, "3-D Ultrasound Imaging", IEEE EMBS, 15-6, pp.41-51, 1996.

(3) A. Matani, M. Nambu, A. Kondo, O. Oshiro and K. Chihara, "A Method of 3D Measurement with High Resolution Using Ultrasound Spherical Waves", Japanese Journal of Applied Physics, 36, pp.3255-3259, 1997.

(4) D. H. Johnson and D. E. Dudgeon, "Array Signal Processing", Eaglewood Cliffs, New Jersey, pp.59-110, 1993.

(5) P. M. Kennedy, D. X. Hammer and B. A. Rockwell, "Laserinduced Breakdown in Aqueous Media", Prog. Quant. Electr., 21, pp.155-248, 1997.

(6) O. Oshiro, M. Nambu, S. Nakatsuka, M. Doi and K. Chihara, "Ultrasound Ring Array Probes for High Resolution Imaging", Transactions of the Institute of Systems, Control and Information Engineers, 13 ,pp.244-249, 2000.

Osamu Oshiro (Member) reveived the $\mathrm{Ph} \mathrm{D}$ degree from

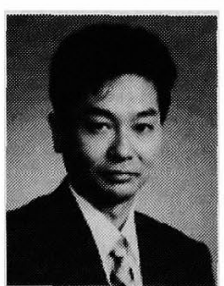
Osaka university in 1990 . He is now the associate professor of Nara institute of science and technology. His current interests are ultrasound measurement, medical imaging and $3 \mathrm{D}$ visualization of a human organ. 
Atsunori Machida (Non-member) received tbe bachelor's

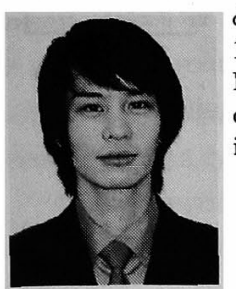
degree from Kyushu institute of technology in 1999. He is now the master course student of Nara institute of science and technology. His current interest is acoustic imaging using laser induced breakdown.
Satishi Kawata (Non-member) received the $\mathrm{Ph} \mathrm{D}$ degree

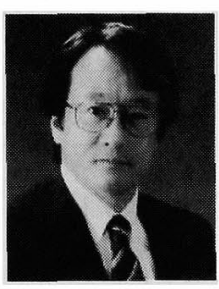
from Osaka university in 1979. He is now the professor of Osaka university. His current interests are near field optics and photonic sensing for biology and medicine.

Motonori Doi (Non-member) received the $\mathrm{Ph} \mathrm{D}$ degree

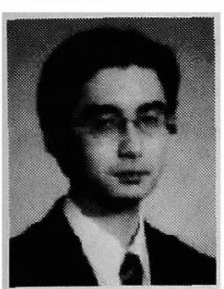
from Nara institute of science and technology in 1998. He is now the assistant professor of Nara institute of science and technology. His current interests are face recognition, medical image analysis and ultrasound sensing.

Kunihiro Chihara (Non-member) received the $\mathrm{Ph} \mathrm{D}$ de-

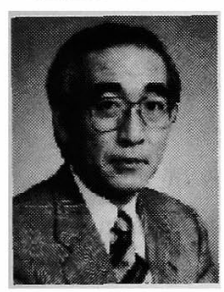
ree from Osaka university in 1973 . He is now the professor of Nara institute of science and technology. His current interests are medical images, image media and virtual museum.

Daisuke Ueda (Non-member) received the bachelor's de-

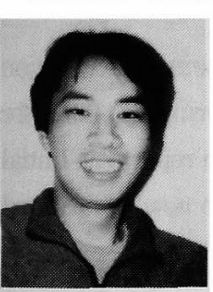
gree from Ritsumeikan university in 1999 . He is now the master course student of Osaka university. His current interest is laser induced breakdown phenomenon.

Tadao Sugiura (Non-member) received the $\mathrm{Ph} \mathrm{D}$ degree

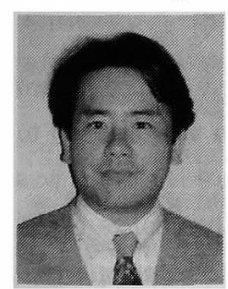
from Osaka university in 1994. He is now the assistant professor of Osaka university. His current interests are photonic sensing for biology and medicine and near field optics. 